\title{
Note on Transcriptions and Citations
}

I have modernized spelling by changing $i$ to $j$ and $u$ to $v$ (and vice versa), but have otherwise retained original spelling and punctuation in all material quoted, unless specified. Passages of Latin and transliterated Greek omit internal accents. All references to classical texts, with the exception of Hermogenes' On Types of Style, are to section numbers rather than page numbers.

Shortened book titles appear in the source citations and footnotes. 